Muséologies

Les cahiers d'études supérieures

muséologies

\title{
Vers une nouvelle typologie des expositions archéologiques
}

\section{Sara Arsenault}

Volume 1, numéro 1, octobre 2006

URI : https://id.erudit.org/iderudit/1033654ar

DOI : https://doi.org/10.7202/1033654ar

Aller au sommaire du numéro

Éditeur(s)

Association Québécoise de Promotion des Recherches Étudiantes en Muséologie (AQPREM)

ISSN

1718-5181 (imprimé)

1929-7815 (numérique)

Découvrir la revue

Citer cet article

Arsenault, S. (2006). Vers une nouvelle typologie des expositions archéologiques. Muséologies, 1(1), 10-25. https://doi.org/10.7202/1033654ar d'utilisation que vous pouvez consulter en ligne.

https://apropos.erudit.org/fr/usagers/politique-dutilisation/ 


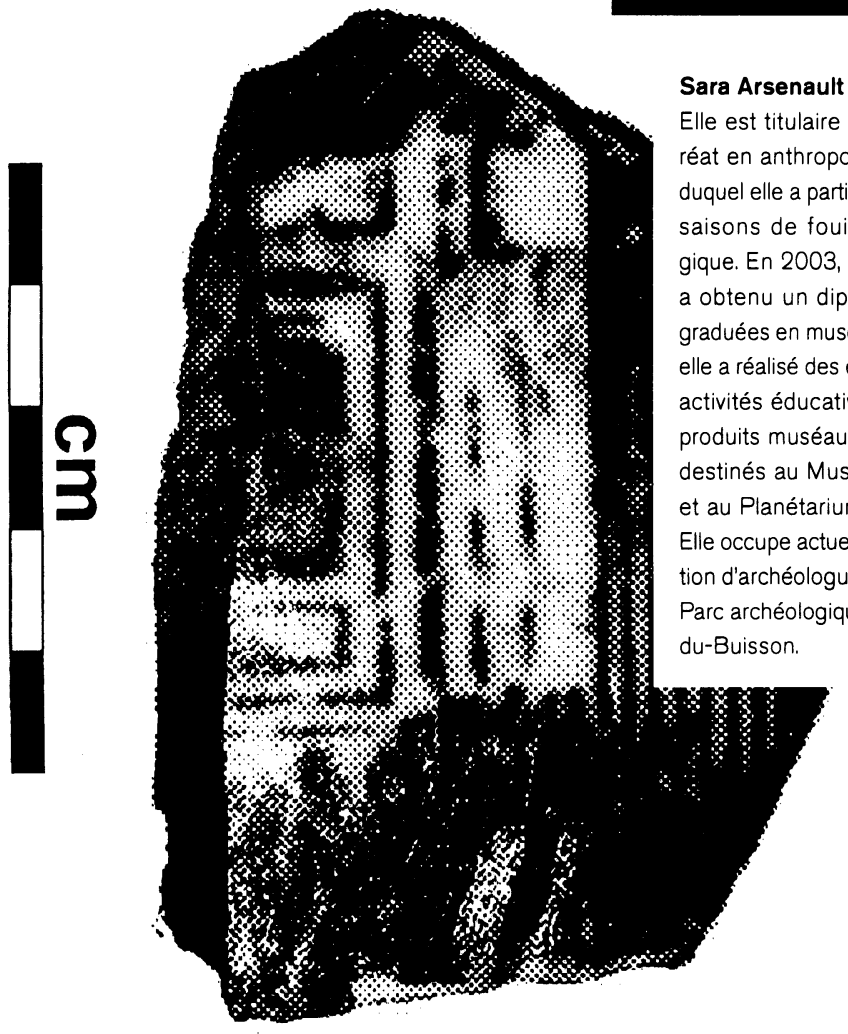

Vers une nouvelle

typologie des

expositions

archéologiques

\section{senault} 2003, Sara Arsenault obtenu un diplôme d'études graduées en muséologie. Depuis, elle a réalisé des expositions, des activités éducatives et d'autres produits muséaux dont certains destinés au Musée de Lachine et au Planétarium de Montréal. Elle occupe actuellement la fonction d'archéologue-animateur au Parc archéologique de la Pointedu-Buisson. 
De prime abord, ce n'est pas dans le contexte muséal que la diffusion de l'archéologie est la plus aisée. De fait, la relation visiteurs-fragments archéologiques ne va pas de soi et faire signifier les témoins matériels constitue un défi. C'est plutôt le site archéologique ${ }^{[1]}$, élément didactique très fort, qui constitue l'interface idéale entre le public et l'archéologie. Cela dit, bien que les lieux de diffusion de l'archéologie au Québec soient de plus en plus nombreux depuis une trentaine d'années, peu d'entre eux offrent la possibilité d'amener les visiteurs sur un chantier de fouilles. La mise en valeur se fait donc principalement par le biais d'expositions.

Dans le cadre d'un travail de fin de maîtrise, une vitrine d'actualités archéologiques a été conçue pour Pointe-à-Callière Musée d'archéologie et d'histoire de Montréal. La réalisation de cette exposition de petite taille était l'occasion idéale de sélectionner une problématique concernant l'arrimage de l'archéologie et de la muséologie pour en faire une étude. C'est le traitement des témoins archéologiques en contexte muséal qui a retenu notre attention.

[1]

DESROSIERS, Pierre. MSL 6521. Muséologie et archéologie, hiver 2002. 
Ce terme est absent de la documentation. Nous nous sommes inspirée de l'expression "muséologie archéologique" avancée par Pothier (1994) POTHIER, Louise. "D'objet à ... sujet. " dans Fragments sous la ville. Les collections archéologiques de Montréal. DUFRESNE, Sylvie (dir.), Pointe-à-Callière, Musée d'archéologie et d'histoire de Montréal, Montréal, 1994, pp.52-55

pour créer ce mot. Nous le proposons afin de qualifier les témoins archéologiques qui sont mis en exposition. Nous reconnaissons un statut particulier aux objets archéomuséaux. Ils sont d'abord des objets de recherche, mais ils possèdent aussi un fort potentiel évocateur et sont mis en valeur précisément pour cette raison.

\section{Problématique}

Une revue des écrits sur la recherche fondamentale en archéologie, les contraintes et les difficultés liées à la réalisation d'une exposition d'archéologie, la médiation avec le grand public et l'objet "archéomuséal ${ }^{[2]}$ " a été réalisée. Ces quatre champs permettent de suivre le passage du témoin archéologique à l'objet muséal. Selon les auteurs consultés, les expositions d'archéologie exemplaires conféreraient un statut particulier aux témoins matériels présentés. Le statut de l'objet archéologique au musée ne devrait pas être celui d'une œuvre d'art ni celui d'un objet strictement technologique. Il doit plutôt être envisagé comme un objet polysémique dans sa culture d'origine. Ce sont les messages de l'objet, le contenu derrière le contenant, que les muséologues devraient privilégier. Cette approche socialisante et humaine de l'artefact en contexte muséal est cohérente avec les objectifs de la recherche archéologique. Si cette approche est privilégiée dans la théorie, est-elle mise en pratique lors de la conception d'expositions?

Nous dressons la liste des paramètres idéaux colligés au cours de notre revue des écrits. Ces paramètres concernent la scénographie, l'interprétation et la médiation des témoins matériels archéologiques.

\section{La scénographie}

Le terme scénographie est employé ici non seulement pour désigner la mise en scène de l'exposition, mais aussi la sélection des artefacts. La stratégie de mise en exposition privilégiée par les auteurs consiste à présenter des objets de petite ou de moyenne taille non isolés les uns des autres. L'aspect esthétique des objets ne doit pas prévaloir, c'est-à-dire que les artefacts exposés n'ont pas à être complets ni réalisés à partir de matériaux nobles. La culture matérielle est présentée dans sa totalité afin d'obtenir une image la plus complète possible de la société passée. Une présentation idéale regrouperait donc des objets façonnés par l'humain et des échantillons naturels, des objets quotidiens autant que des objets de valeur.

Les objets archéologiques sont muets, ils ne parlent pas d'eux-mêmes. Ainsi, ils sont obligatoirement accompagnés de supports didactiques. Au minimum, un cartel d'identification doit comporter le nom de l'objet et sa matière. Il est fortement recommandé d'associer des textes explicatifs aux objets. Une exposition d'archéologie modèle fournirait ce second niveau de lecture de l'objet.

\section{L'interprétation}

Sachant que les artefacts doivent être accompagnés de textes explicatifs, il convient ensuite de se questionner sur ce qui doit être dit à propos des objets archéologiques. Au-delà des attributs intrinsèques des objets, les auteurs recommandent que les attributs extrinsèques soient évoqués. Il est souhaitable 
d'aborder le groupe humain, les comportements, les idéologies et les valeurs sociales liés à l'artefact. C'est la signification culturelle de l'objet qui doit être mise de l'avant et non le travail de terrain de l'archéologue. En effet, l'objectif de l'exposition d'archéologie modèle n'est pas de promouvoir et de légitimer la recherche archéologique, mais bien de faire découvrir les cultures passées. Une exposition réussie doit permettre aux visiteurs de percevoir l'humain producteur ou utilisateur derrière l'artefact.

\section{La médiation}

Quatre principes directeurs permettent d'optimiser la rencontre entre le visiteur et les fragments archéologiques. D'abord, les interprétations doivent être justifiées et expliquées. Par exemple, dans l'exposition Soudan : royaumes surle Nil analysée par O'Neill, le commissionnaire ne s'est pas limité à présenter un outil simplement comme une masse non usée. L'explication suivante était donnée : cette masse n'est pas usée parce que c'est un objet rituel ${ }^{[3]}$. Ainsi, des renseignements sont livrés au visiteur afin qu'il joue mentalement avec l'objet, qu'il l'interprète et applique ce raisonnement à d'autres objets. Cette démarche s'inscrit dans le courant de pensée néo-muséologique qui veut créer un sentiment de valorisation chez le visiteur; ce dernier doit en venir à considérer le musée comme un lieu accessible. Aussi, les interprétations présentées doivent-elles être ouvertes et multiples pour impliquer le public, pour lui permettre de participer au raisonnement de l'archéologue et de forger sa propre opinion. Évidemment, des textes rédigés dans un langage simple et

TABLEAU 1: Grille d'analyse du statut de l'objet archéomuséal en trois grands domaines

[Scénographie]

\begin{tabular}{|c|c|}
\hline Variable & Observations \\
\hline $\begin{array}{l}\text { Taille des témoins } \\
\text { archéologiques exposés }\end{array}$ & $\begin{array}{l}\text { 1] la proportion des objets monumentaux } \\
\text { est plus importante } \\
\text { 2] les objets de petite ou de moyenne taille } \\
\text { sont majoritaires }\end{array}$ \\
\hline Mise en vitrine des objets & $\begin{array}{l}\text { 1] certains objets sont mis sur un piédestal, } \\
\text { ils sont isolés des autres } \\
\text { 2] les objets sont regroupés et traités sur un même plan }\end{array}$ \\
\hline État des artefacts & $\begin{array}{l}\text { 1] les témoins matériels complets prédominent } \\
\text { 2] les objets fragmentaires sont majoritaires }\end{array}$ \\
\hline Matériaux & $\begin{array}{l}\text { 1] la plupart des objets sont réalisés à partir } \\
\text { de matériaux nobles } \\
\text { 2] les artefacts sont principalement exécutés } \\
\text { dans des matériaux communs }\end{array}$ \\
\hline
\end{tabular}

\section{[3]}

O'Neill, MARIE-CLARTÉ. "Le discours d'une exposition: identification, explication, implication. In. DUFRESNE-TASSÉ, Colette (éd.). Évaluation et éducation muséale : nouvelles tendances / Evaluation and Museum Education: New Trends / Evaluación y Educación de los Museos: Nuevas Direcciones. Paris : Conseil International des musées de I'UNESCO, 1998. p. 77-87. 


\section{TABLEAU 1: suite}

\section{[Scénographie] suite}

\section{Variable}

Nature des objets

1] seuls des artefacts, c'est-à-dire des objets façonnés ou altérés par l'intervention humaine, sont présentés

2] des écofacts, vestiges d'origine naturelle, sont aussi exposés

Catégorie des artefacts 1] insistance sur les objets de valeur que la majorité ne possédait pas

2] les objets quotidiens sont majoritaires

Supports didactiques 1] absence de support didactique

2] des cartels et/ou des commentaires explicatifs sont associés aux objets exposés

[Interprétation]

\section{Variable}

Sujet principal de l'exposition

Caractéristiques intrinsèques des artefacts

L'humain au centre de la présentation

Signification culturelle des objets

\section{Observations}

1] la description du travail de terrain de l'archéologue fait partie intégrante du discours

2] l'accent est mis sur la présentation d'une culture passée

1] les objets sont peu décrits

2] maintes caractéristiques matérielles sont évoquées

1] l'objet semble plus important que l'humain

2] des renseignements ou des hypothèses sont proposés au sujet de l'humain producteur, propriétaire et/ou utilisateur de l'objet

1] le contexte culturel dans lequel les artefacts faisaient sens n'est pas évoqué

2] il est question des comportements et des modes de vie liés aux artefacts et/ou des valeurs et des idéologies qu'ils traduisent

\section{[Médiation ]}

\section{Variable}

\section{Observations}

Explication

1] les explications sont absentes ou rarissimes

2] les résultats et les conclusions des chercheurs sont expliqués au public, ils ne sont pas présentés comme des évidences

Implication

1] la réflexion et le questionnement ne sont pas encouragés

2] les interprétations sont ouvertes et multiples, ce qui permet au visiteur de participer au raisonnement et de se faire sa propre idée

Démocratisation

1] le niveau de langage ne convient pas au public non spécialiste

2] les textes sont écrits dans un langage accessible et cohérent afin de n'exclure aucun public

Mise à profit de l'émotion 1] le contexte culturel dans lequel les artefacts faisaient sens n'est pas évoqué

2] il est question des comportements et des modes de vie liés aux artefacts et/ou des valeurs et des idéologies qu'ils traduisent 
cohérent assurent que les messages de l'exposition sont communiqués à tous les publics. Finalement, une exposition d'archéologie réussie fait appel à la sensibilité des gens et à leurs savoirs émotifs. Elle ne se contente pas de tirer profit des savoirs scientifiques.

\section{Grille d'analyse du statut de l'objet}

Les textes consultés ont fourni les critères de base à l'élaboration de la grille d'analyse du statut de l'objet archéomuséal, qui comprend quinze variables opératoires réparties entre les sphères de la scénographie, de l’interprétation et de la médiation de l'objet.

\section{Méthodologie}

Pour chacune des variables étudiées, il s'agissait de déterminer lequel des niveaux proposés s'applique à la mise en valeur archéologique. La grille d'analyse a été appliquée à la vitrine archéologique que nous avons conçue, Louis-Hector de Callière militaire et diplomate ${ }^{[4]}$ [FIGURE 1], ainsi qu'à deux autres présentations : d'abord aux vitrines intitulées Des objets, des populations [FIGURE 2], qui constituent la première section de l'exposition de longue durée de Pointe-à-Callière ${ }^{(5)}$, ensuite à la Salle des découvertes [FIGURE 3 ] du Musée Marguerite-Bourgeoys.

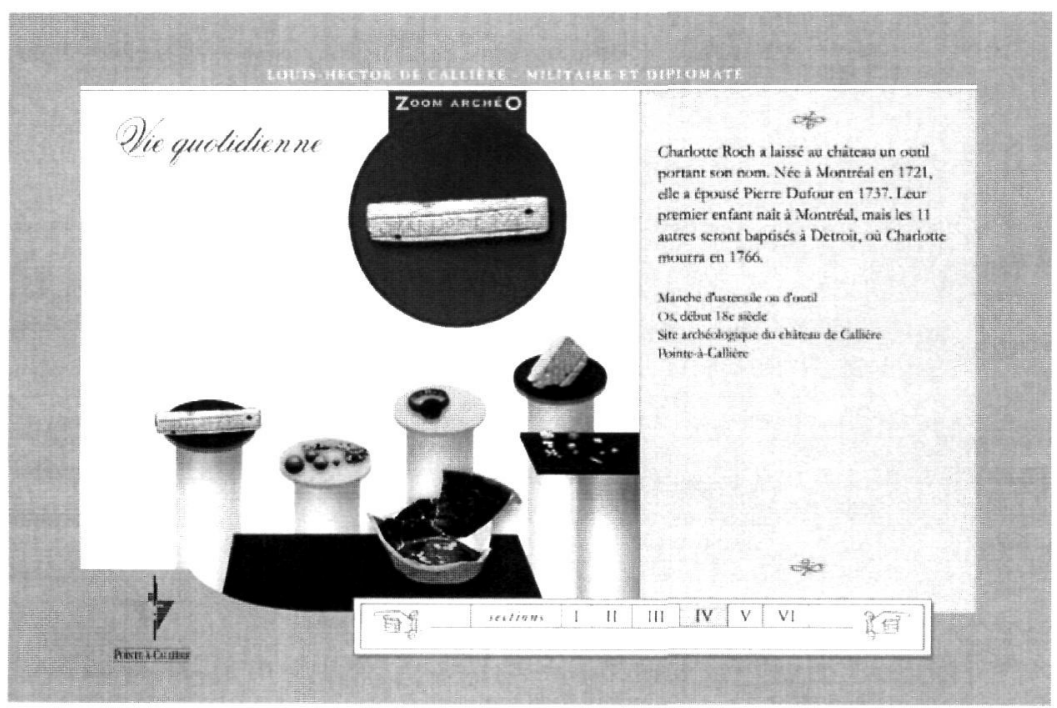

FIGURE 1 .

Quelques objets présentés dans la vitrine Louis-Hector de Callière, militaire et diplomate. Image tirée de l'exposition virtuelle sur le même sujet. @ Pointe-à-Callière, Musée d'archéologie et d'histoire de Montréal [ Adaptation Web : Maristella Designer Multimédia ]
[4]

La vitrine d'actualités était une présentation muséale temporaire. On peut accéder au contenu et au concept de la vitrine sur le site <http://www. pacmusee.qc.ca/actualites_ archeo/lhcalliere_fr/default. htm>.

[5]

En mai 2003, après dix ans d'existence, Pointe-à-Callière, Musée d'archéologie et d'histoire de Montréal a renouvelé son exposition de longue durée. La section Des objets, des populations est maintenant différente de celle analysée dans cette étude. 
Nous avons retenu ces trois situations parce qu'elles sont comparables en plusieurs points. Premièrement, ces mises en valeur sont de taille restreinte : la quantité d'information communiquée est semblable. Les mises en valeur choisies concernent des fouilles archéologiques qui ont eu lieu dans la même portion de la ville (le Vieux-Montréal) et qui ont livré des vestiges datant d'époques identiques, soit les deux premiers siècles d'existence de Montréal. De plus, dans les trois cas, les musées se sont associés au projet de fouilles. Il est donc légitime de croire que l'accès aux données archéologiques était optimal lors de la conception des mises en valeur. Finalement, les musées retenus pour cette analyse ont une mission semblable.

[6]

AMYOTTE, LUC

Méthodes quantitatives: Application à la recherche en sciences humaines. SaintLaurent : Éditions du renouveau pédagogique inc., 1996. 480.

p. 4-14.

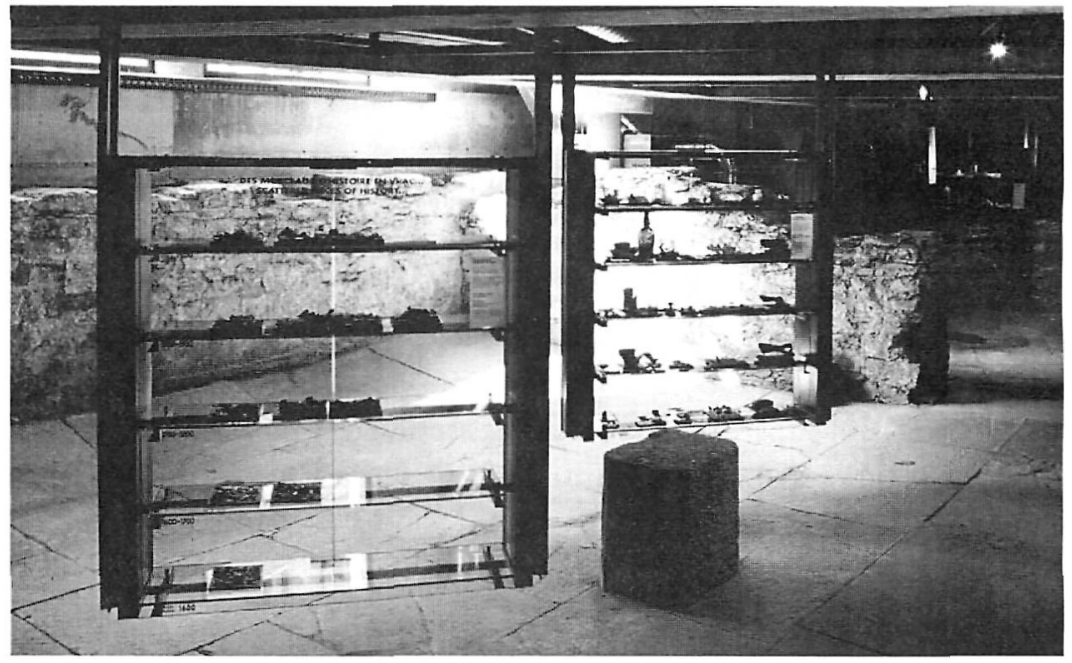

FIGURE 2.

Deux des présentoirs de la mise en valeur Des objets, des populations dans l'exposition permanente de Pointe-à-Callière, Musée d'archéologie et d'histoire de Montréal. @ Pointe-à-Callière, Musée d'archéologie et d'histoire de Montréal [ Photographe : Roderick Chen]

La grille d'analyse a été appliquée aux mises en valeur choisies. Nous avons procédé à l'analyse du statut de l'objet par observation directe des présentations et par l'étude des documents muséologiques associés (concepts, scénarios, etc.). Afin d'assurer l'impartialité des résultats, nous avons demandé à trois collègues d'évaluer la vitrine que nous avons réalisée. Cette méthode a l'avantage de déterminer la validité de la grille d'analyse, les résultats devant être reproductibles pour une même présentation muséale ${ }^{[6]}$.

L'application de cette grille d'évaluation permet de vérifier si le statut de l'objet archéologique est identique dans les trois expositions. Malgré la définition d'un statut idéal de l'objet archéomuséal dans les publications, notre hypothèse de départ est la suivante : l'approche de l'artefact n'est pas la même dans chaque mise en valeur et le traitement de l'objet archéologique n'est pas idéal. 


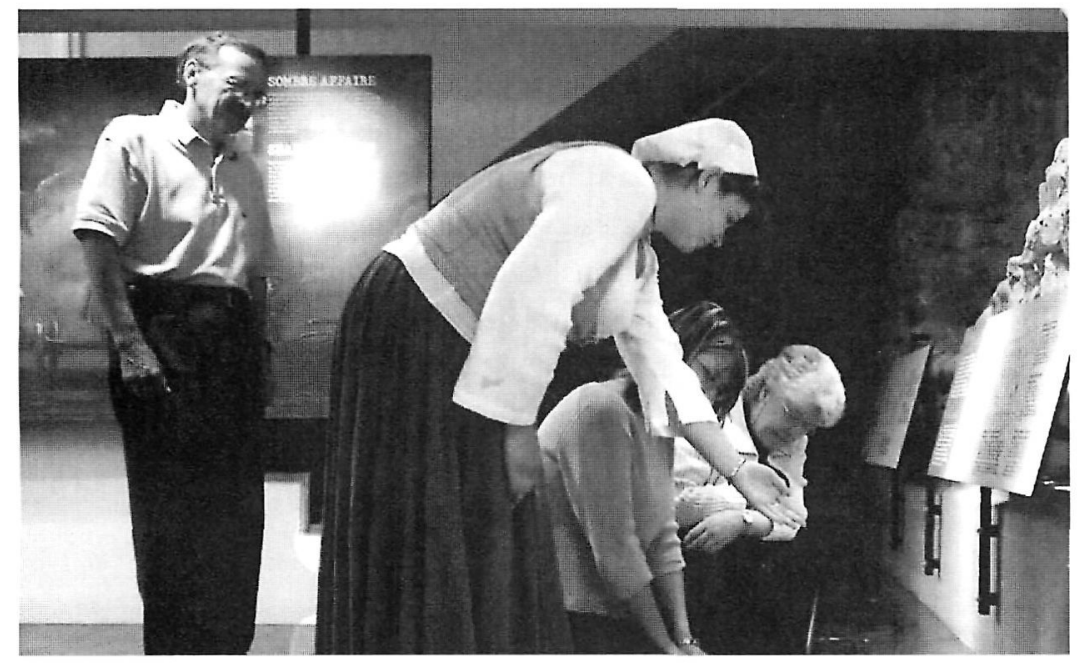

FIGURE 3.

Portion de la Salle des découvertes du Musée Marguerite-Bourgeoys. @ Musée Marguerite-Bourgeoys [Photographe : Bernard Dubois]

\section{Le statut de l'objet archéomuséal}

Bien que cette recherche soit de nature qualitative, nous croyons pertinent de quantifier les résultats afin de les comparer à un premier niveau. Un système de notation mesure le respect des principes de présentation, d'interprétation et de médiation des témoins archéologiques. Pour chaque variable opératoire de la grille, un point est attribué à la mise en valeur si les observatrices ont retenu le niveau recommandé par les auteurs sur la muséologie archéologique. Un pointage nul signifie que les réalisations dans ce secteur ne sont pas conformes aux préceptes rencontrés dans la revue des écrits. Pour chacune des mises en valeur choisies, nous avons compilé les pointages selon les trois sphères à l'étude .

\section{TABLEAU 2 :}

Tableau présentant les pointages des mises en valeur selon les sphères étudiées

\begin{tabular}{|c|c|c|c|}
\hline & $\begin{array}{l}\text { Des objets, } \\
\text { des populations }\end{array}$ & $\begin{array}{l}\text { Salle des } \\
\text { découvertes }\end{array}$ & $\begin{array}{l}\text { Vitrine d'actualités } \\
\text { archéologiques }\end{array}$ \\
\hline Scénographie & $7 / 7$ & $7 / 7$ & $6 / 7$ \\
\hline Interprétation & $2 / 4$ & $2 / 4$ & $4 / 4$ \\
\hline Médiation & $3 / 4$ & $3 / 4$ & $3 / 4$ \\
\hline Total & $12 / 15$ & $12 / 15$ & $13 / 15$ \\
\hline
\end{tabular}


Il est important de rappeler que ces données ne sont présentées qu'à titre indicatif et non comme des données statistiques formelles. Néanmoins, la comparaison de ces pointages révèle quelques éléments intéressants. D'abord, la scénographie est la sphère la moins problématique alors que l'interprétation et la médiation idéales semblent plus difficiles à atteindre. En effet, la scénographie répond à tous les critères retenus deux fois sur trois, alors que l'interprétation n'est exemplaire qu'en une seule occasion et que la médiation ne l'est en aucun cas. Dans le graphique suivant [figure 4], les fractions ont été converties en pourcentage.

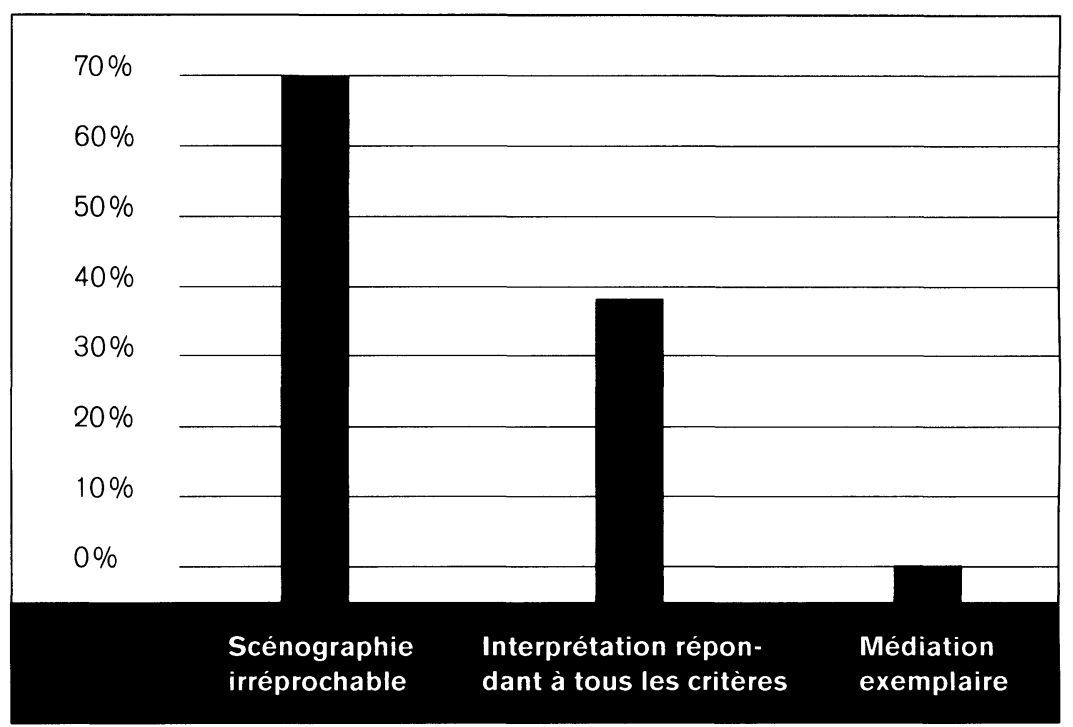

FIGURE 4.

Fréquence des pointages parfaits selon la sphère étudiée

Les concepteurs d'expositions archéologiques semblent maîtriser la mise en scène des témoins. Toutefois, des améliorations devraient être apportées afin de faire parler davantage les objets et d'instaurer une relation interactive avec le visiteur. Cet état des choses n'est pas surprenant puisqu'il est plus simple pour une institution muséale d'adopter une muséographie dans laquelle les artefacts ne sont pas envisagés comme des œuvres d'art que d'explorer en profondeur l'interprétation des objets et de rendre plus dynamique le rapport entre ces derniers et le public.

Si la scénographie a été décrite comme très satisfaisante dans les trois expositions visitées, nous croyons qu'il n'en est pas toujours ainsi. Nous serions probablement confrontée à une scénographie désuète en étudiant le traitement des artefacts dans des expositions conçues par des établissements muséaux où l'archéologie ne serait pas la discipline principale. 
Ensuite, c'est sur le plan de l'interprétation que les pointages des mises en valeur sont les plus variables. Cela démontre qu'il est exigeant de faire signifier les artefacts. À ce propos, Lacroix fournit une explication à laquelle nous adhérons. Il affirme que peu d'institutions possèdent les ressources humaines et financières pour documenter les objets en profondeur lors de leur acquisition : «Les musées accumulent sans avoir toujours identifié le potentiel de ces objets. Certes, [...] ils disposent d'un cadre de référence qui permet de les classer, mais non de les interpréter $"{ }^{[7]}$.

Il faut faire un dernier constat général : aucune des expositions analysées n'est parfaite, mais aucune n'est médiocre non plus. Elles répondent toutes à la majorité des critères établis, soit 12/15 pour Des objets, des populations et la Salle des découvertes, et 13/15 dans le cas de la vitrine d'actualités archéologiques. Malgré ces pointages semblables, il ne faut pas croire que ces mises en valeur soient équivalentes. Les expositions ont leurs forces et leurs faiblesses respectives, ce qui tend à confirmer l'existence de plusieurs approches de l'objet archéomuséal au sein des musées d'archéologie.

\section{Les différentes approches de l'objet archéomuséal}

Un résumé des particularités des trois mises en valeur étudiées nous permet d'esquisser une typologie des expositions archéologiques quant à leur traitement de l'objet archéomuséal.

Des objets, des populations, Pointe-à-Callière, Musée d'archéologie et d'histoire de Montréal.

Selon les données recueillies sur le terrain et l'information colligée dans les documents muséaux, trois recommandations des auteurs sur la muséologie archéologique n'ont pas suffisamment été prises en compte lors de la conception de cette exposition. Des objets, des populations a d'abord sa faiblesse propre, soit le manque d'information sur les utilisateurs et les autres individus associés aux histoires de vie des objets. Cette dimension n'a pas été privilégiée et ce qui importe ici davantage est la présentation d'objets significatifs pour une époque donnée. Les deux autres points qui mériteraient d'être améliorés sont la description précise des artefacts et l'implication du visiteur, mais ces deux éléments sont aussi insatisfaisants dans d'autres expositions étudiées.

Bien que Des objets, des populations rencontre la majorité des critères établis, nous estimons que cette exposition ne s'illustre pas comme étant exceptionnelle dans aucun domaine. Effectivement, même si nous avons jugé le traitement de l'objet archéomuséal approprié en plusieurs points, nous constatons que le rendement est inférieur à celui des autres expositions examinées. Par exemple, des cartels sont associés à chacun des objets, mais il y a absence totale de commentaires explicatifs alors que certains ont été XXle siècle». Muse. vol. 17, no 2, 1999. p. 6-10. 
inclus dans la vitrine d'actualités archéologiques et dans la Salle des découvertes. De même, la signification culturelle des objets est mise de l'avant en de plus rares occasions et de manière moins explicite. Le même constat peut être fait à propos de l'explication du raisonnement des chercheurs.

Ainsi, cette mise en valeur est dite généraliste car elle satisfait la majorité des critères bien qu'aucun domaine du statut de l'objet archéomuséal (la scénographie, l'interprétation ou la médiation) ne soit développé en profondeur.

Salle des découvertes, Musée Marguerite-Bourgeoys

L'implication du visiteur est particulièrement bien développée dans cette salle d'exposition. En fait, c'est l'ensemble de la relation établie entre les artefacts et le public qui est supérieure à celle observée dans les autres mises en valeur, même si les trois expositions obtiennent un pointage égal dans le domaine de la médiation de l'objet archéomuséal (soit trois sur quatre). Nous croyons que la variable jugée inadéquate dans la Salle des découvertes, l'accessibilité du texte, est moins importante que l'implication du visiteur jugée déficiente dans les deux expositions de Pointe-à-Callière. Les points forts que sont l'intégration du public au raisonnement archéologique et l'explication des conclusions des chercheurs n'apparaissent pas exceptionnels dans notre grille d'analyse. En effet, il résulte un important manque de nuance dans le choix d'une grille de type présence-absence : les éléments simplement satisfaisants ne sont pas discriminés de ceux qui sont exemplaires.

Cela dit, la Salle des découvertes ne se distingue pas seulement par ses forces, elle a aussi quelques faiblesses. D'abord, les références au travail de terrain des archéologues sont fort nombreuses et détaillées. Le fait que l'information relative aux recherches archéologiques soit présentée sous forme de manchettes journalistiques démontre bien que l'exposition mise sur l'intérêt de la population pour les fouilles. L'évocation du travail de terrain des archéologues ne devrait pas être traitée de façon détaillée dans la salle d'exposition. Les artefacts ont un potentiel de représentation immense; ils symbolisent des modes de vie, des comportements et des valeurs et ils doivent avant tout être exploités à cette fin. Les supports didactiques mériteraient d'être standardisés. Ces derniers sont idéaux pour un grand nombre d'objets et totalement inexistants pour d'autres.

En bref, la Salle des découvertes est une exposition que nous qualifions d'archéomédiative parce que les concepteurs ont voulu établir un contact très particulier avec le public et parce qu'ils accordent une grande importance à la science archéologique. Le visiteur est invité à devenir un participant actif en intégrant le raisonnement archéologique pour ensuite l'appliquer par luimême au cours de sa visite. Cette mise en valeur se distingue donc des autres par sa pédagogie : un ensemble de questions et d'enseignements est efficacement partagé avec les visiteurs. 
Louis-Hector de Callière : militaire et diplomate, Pointe-à-Callière

Nos collègues ont noté deux principales lacunes quant au statut de l'objet archéomuséal dans cette mise en valeur : la première concerne la scénographie et la seconde, la médiation. D'abord, aucun écofact n'a été mis en exposition, contrairement à ce qui est recommandé dans les publications. Dans le concept original, les graines de fruits trouvées en association avec une terrine devaient être exposées. Malheureusement, ces témoins d'origine naturelle n'ont pas été intégrés à la collection du musée : ils ont été aliénés. Tous les vestiges exhumés, catalogués et analysés par les archéologues n'entrent pas en bloc dans la collection. Une certaine sélection est pratiquée. Cela semble confirmer qu'une valeur moindre est attribuée aux écofacts et explique en partie le fait qu'ils soient plus rarement mis en exposition. Ensuite, le second point faible est la non-implication du visiteur. Les renseignements portant sur les artefacts ne sont pas présentés de manière à inciter le public à se questionner à leur sujet et à formuler des hypothèses personnelles.

Malgré ces deux critiques, la vitrine d'actualités archéologiques se démarque des autres mises en valeur par son interprétation probante des artefacts. En premier lieu, la description des objets archéologiques est complète et standardisée. Ensuite, des renseignements fort nombreux et précis sont dévoilés à propos des humains liés aux histoires de vie des objets et de la signification culturelle des artefacts. Les objets exposés ont été sélectionnés parce qu'ils étaient particulièrement parlants relativement à la vie de LouisHector de Callière et aux diverses occupations de sa résidence.

La vitrine Louis-Hector de Callière, militaire et diplomate met principalement l'accent sur l'interprétation des témoins archéologiques, bien que la scénographie et la médiation soient légèrement déficientes. Nous qualifions d'interprétatives les expositions dans lesquelles les concepteurs s'efforcent de replacer les objets dans leur contexte d'origine et de dévoiler leur signification.

\section{Comparaison des résultats avec d'autres typologies muséales}

Cette étude permet de définir une nouvelle typologie des expositions archéologiques composée des mises en valeur dites généralistes, archéomédiatives et interprétatives. La typologie proposée demeure inachevée et devra être raffinée, mais elle apparaît tout de même pertinente. Elle complète d'autres modèles de classement des expositions au sein desquels les trois mises en valeur étudiées entrent systématiquement dans les mêmes catégories. Nous pensons ici aux typologies de Davallon, d'Ettema et de Pomian.

En 1986, Davallon ${ }^{[8]}$ établit une typologie des expositions. Dans ce premier classement, il définit trois grandes catégories de mise en valeur. D'abord,
[8]

DAVALLON, Jean.

"Avant-propos *. In. DAVALLON, Jean (dir.). Claquemurer pour ainsi dire tout l'univers. Paris : Centre Georges-Pompidou, 1986. p. 7-16. 
MONTPETIT, Raymond Dans le cours MSL6104.

Exposition, interprétation et diffusion, Université du Québec à Montréal, hiver 2002.

[10]

ETTEMA, Michael J. "History Museums and the Culture of materialism " In. BLATTI, Jo (dir.). Past Meets Present. Essays about Historic Interpretation and Public Audiences. Washington, D.C. Smithsonian Institution Press, 1987. p. 62-85.

[11]

POMIAN, Krzystof

"Musée archéologique: Art, nature, histoire" Le débat, no 49, 1988. p. 57-68. la muséologie d'objets, qui propose une rencontre entre le visiteur et des objets authentiques. Ce type de présentation correspond à ce que d'autres appellent la muséologie traditionnelle. Il établit ensuite que certaines expositions sont davantage communicationnelles, c'est-à-dire qu'elles visent à faire comprendre une didactique ou un savoir ; l'objectif consiste à transmettre des messages, des connaissances et des idées. Finalement, il avance que d'autres expositions visent un impact social en tentant d'éveiller chez le visiteur un sentiment d'appartenance. Au cours de la dernière décennie, Davallon a revu cette classification à deux reprises ${ }^{[0]}$, mais ces modifications ne sont guère pertinentes à notre propos puisque les mises en valeur étudiées entrent toutes dans la deuxième catégorie de la typologie originale, soit la muséologie d'idées.

La classification d'Ettema ${ }^{[10]}$ ''adresse cette fois directement aux expositions d'archéologie. Selon cet auteur, les artefacts constituent le centre d'intérêt de l'approche formaliste : ils sont identifiés en termes d'époque, de fonction, de provenance et d'évolution stylistique. À l'opposé, on trouve la perspective analytique. Les artefacts sont utilisés pour stimuler des questions et communiquer plus facilement des idées. Ils ne sont pas le centre de la présentation. La perspective analytique communique des concepts qui permettent au public de formuler des explications à propos des modes de vie des sociétés passées. Les concepteurs des trois mises en valeur montréalaises analysées ont opté pour la stratégie analytique.

Pomian [11] identifie deux types de musées archéologiques. Le musée archéo-artistique présente des objets exécutés à partir de matériaux nobles; ils sont généralement de grande dimension et isolés les uns des autres. Les artefacts sont en fait présentés comme des œuvres d'art qui parlent d'ellesmêmes. Le musée archéo-technologique, pour sa part, expose des objets de taille réduite en les regroupant. Les cartels sont complétés par des textes, des plans et des photographies. Ces supports didactiques permettent de replacer les artefacts dans leur contexte d'origine. En plus des objets façonnés par l'humain, ce type de musée présente des écofacts, rejetant la coupure entre la nature et la culture. Cette approche dite universelle s'intéresse à la vie quotidienne de toutes les strates de la société et à toutes les époques. Les expositions étudiées à Pointe-à-Callière et au Musée Marguerite-Bourgeoys sont définitivement de type archéo-technologique.

Les typologies de Davallon, d'Ettema et de Pomian ne permettent pas de particulariser les expositions étudiées dans cette étude. La typologie que nous proposons a l'avantage de les distinguer et s'applique probablement à plusieurs autres mises en valeur. 


\section{Perspectives}

Pour faire suite à cette étude, nous désirons soulever quelques critiques concernant la conception et l'application de notre grille d'analyse du statut de l'objet archéomuséal. D'abord, la mise à profit de l'émotion, qui est une des variables de la grille, n'est pas suffisamment explicite. Les deux niveaux proposés pour qualifier cette variable laissent place à l'interprétation. Nous proposons de reformuler les deux niveaux comme suit :

1] La présentation réfère uniquement au savoir scientifique. L'exposition ne consiste qu'en la présentation de faits.

2] L'exposition suscite des émotions. Par exemple, le visiteur est étonné, ému, choqué ou fier.

Ensuite, une trop grande importance est accordée à la scénographie par rapport aux autres dimensions : sept variables définissent cette sphère contre quatre pour chacune des autres. Ce déséquilibre est d'autant plus problématique que la scénographie nous apparaît une dimension moins fondamentale que l'interprétation et la médiation par lesquelles l'exposition transmet les connaissances et le raisonnement archéologiques. Pour remédier à ce problème, il suffit de regrouper des couples de variables qui sont fortement corrélées. Par exemple, puisque les objets de la vie quotidienne sont presque toujours construits dans des matériaux communs et que les pièces de valeur sont faites de matières premières nobles, les paramètres que sont la catégorie des objets et le matériau pourraient être combinés. Il en va de même de la taille des objets et de leur état de conservation : ces variables auraient avantage à être fusionnées.

\section{Conclusion}

Lors de la formulation de notre problématique, nous estimions que l'approche de l'artefact ne serait pas la même dans chacune des mises en valeur étudiées et que le statut de l'objet archéomuséal serait loin d'être idéal. Selon notre analyse, la première hypothèse est confirmée alors que la seconde est rejetée.

Comme nous l'avions escompté, il semble exister différentes stratégies liées au traitement de l'artefact au sein des musées d'archéologie. Les trois expositions analysées correspondent à trois approches distinctes. Il y a d'abord les mises en valeur dites généralistes qui ne développent spécifiquement aucun domaine du statut de l'objet archéomuséal, mais qui répondent tout de même à la majorité des critères. Les expositions archéomédiatives, quant à elles, s'attachent à diffuser le raisonnement archéologique et à permettre aux visiteurs d'y participer. Finalement, les expositions interprétatives se 
concentrent sur l'évocation des comportements, des modes de vie, des événements et des individus que nous révèlent les artefacts.

Au regard de notre seconde hypothèse, il semble que le statut de l'objet archéomuséal soit satisfaisant dans l'ensemble des expositions étudiées, contrairement à ce que nous avions avancé au départ. Nous pensions que les recommandations des auteurs sur la muséologie archéologique demeuraient essentiellement théoriques et ne seraient guère appliquées dans la pratique. Nous étions d'avis qu'il y aurait des écarts considérables entre les résultats des trois mises en valeur. Ces suppositions sont infirmées : les expositions obtiennent des pointages semblables même si le potentiel des objets archéologiques n'est pas développé avec la même intensité dans chacune des mises en valeur.

L'archéologie et la muséologie sont, au Québec, des disciplines relativement nouvelles. Il y a fort à parier que leur arrimage se raffinera au cours des prochaines décennies.

\section{Summary}

\section{Towards a new archeaological exhibition typology}

The conception of a small-scale archaeological exhibition during her Master's in Museum Studies provided Sara Arsenault with the chance to study the anchoring of archaeology and museology, in particular the treatment of archaeological evidence in a museum context. A review of the literature on the subject allowed for a definition of the ideal status of the archaeo-museological object. Exemplary archaeological exhibitions confer a particular status to the materials presented. The status of the archaeological object in museums should not be that of a work of art nor that of a strictly technological object. Rather, it should be seen as a polysemic artefact in its culture of origin. If this socializing approach is privileged in theory, it must be asked whether it is indeed put into practice during the conception of exhibitions.

In order to evaluate the display, interpretation and mediation of archaeological objects in museums, an analytical grid was conceived. The author is therefore interested in the ways in which exhibition developers decide to present artefacts, in the messages they deliver to visitors, and in the relationship that is established between the latter and the objects.

The grid was applied to three archaeological exhibitions in Montreal, at Pointe-àCallière, Montreal Museum of Archeology and History and at the Marguerite-Bourgeoys 
Museum. The author presented the following hypothesis: the artefact approach is not the same in each display and the treatment of the archaeological object is not ideal.

According to the results obtained, the display seems to be the least problematic element. Ideal interpretation and mediation seem more difficult to attain. In fact, the scenography fulfills grid criteria two times out of three, whereas the interpretation is exemplary on only one occasion and mediation fails completely. Nevertheless, all the presentations that were studied obtained a satisfactory evaluation, which seems to support the hypothesis that the archaeo-museological object is not suitably exhibited.

There are many ways of exhibiting archaeological objects in museums. The exhibit Des objets, des populations at Pointe-à-Callière Museum is said to be generalistic because no area of the archaeo-museological object status is further developed. The Salle des découvertes of the Marguerite-Bourgeoys Museum follows an archaeomediative approach since it is committed to the diffusion of archaeological reasoning and to enabling visitor-participation. Finally, the Louis-Hector de Callière: militaire et diplomate showcase is interpretive because the artefacts evoque human behaviour, ways of life and events. This new typology is distinct from those proposed in recent years by Davallon, Ettema and Pomian. In conclusion, proposal of improvements are suggested for the analytical grid devised for the evaluation of the status of the archaeo-museological object. 